\title{
N.G.M. Orie, der Vater der „Dutch Hypothesis“, wurde 91 Jahre alt
}

\author{
N.G.M. Orie, the Father of the "Dutch Hypothesis", Died at Age 91
}

Autor

Institut

\section{G. Schultze-Werninghaus}

Berufsgenossenschaftliche Kliniken Bergmannsheil, Ruhr-Universität Bochum eingereicht 29. 5. 2006

akzeptiert 17. 7.2006

\section{Bibliografie}

DOI $10.1055 / \mathrm{s}-2006-944290$

Pneumologie 2006; 60; 629-631

(c) Georg Thieme Verlag KG

Stuttgart · New York

ISSN 0934-8387

Korrespondenzadresse

Prof Dr. med. Gerhard Schultze-Werninghaus

Berufsgenossenschaftliche Kliniken Bergmannsheil Ruhr-Universität Bochum Medizinische Klinik III Pneumologie, Allergologie, Schlaf- und Beatmungsmedizin Bürkle-de-la-Camp-Platz 1 44789 Bochum gerhard.schultze-werninghaus@rub.de
„Dick“ (Nicolaas Gerardus Maria) Orie, emeritierter Professor für Lungenkrankheiten der Reichsuniversität Groningen, ist im 91. Lebensjahr am 5. Juli 2006 in Winsum/Niederlande verstorben. Professor Orie ist einer der Väter der modernen Pneumologie. Er hat frühzeitig die entzündlichen Aspekte der chronischen obstruktiven Atemwegserkrankungen $\mathrm{zu}$ seinem Forschungsschwerpunkt gemacht und damit die moderne Forschung über die Grundlagen von Asthma und COPD begründet.

Der Leitgedanke von Orie u. Mitarb. in Groningen war um 1960, dass - neben exogenen Faktoren patienteneigene Eigenschaften („host factors“), insbesondere die Bereitschaft für die Entzündung von Atemwegen und Alveolen, messbar in der bronchialen Hyperreagibilität, für die Entstehung von Asthma und COPD gleichermaßen bedeutsam seien (O Abb. 1, 2).

Das Konzept wurde erstmals auf dem Symposium „Bronchitis“ 1960 vorgestellt [3]. Als Schlussfolgerung der Untersuchungen wurde vorgetragen: "The sum of basic disturbances: hyperreactivity and allergy, sequelae such as infection and fibrosis and complicating factors explain to a considerable extent the clinical picture of bronchitis and asthma - and their analysis suggests that bronchitis and asthma are both different patterns of the same conditions whose signs and symptoms are also influenced by age and sex. - It does not explain the "why" of the basic disturbances but it gives us a lead to the direction of further investigations. - Many apparently paradoxical facts: levels of eosinophilia, influence of infection, lifecycles, 24-hour-rhythm (natural history in relation to age and sex) as well as therapeutical observations can be understood in terms of pituitary-adrenal activity. - Much work will however have to be done before this is proved and before the quantitative and qualitative details of its mechanism have been elucidated."

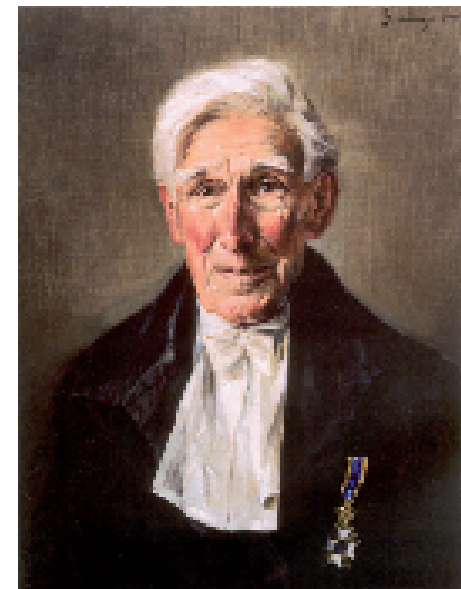

Abb. 1

N. G. M. Orie (1914-2006) J. M. C. Asselbergs, 2002; (c) Reichsuniversität Groningen, Universitätsmuseum

Diese Auffassung stieß auf Widerstände insbesondere im angelsächsischen Raum, da dort Asthma und COPD als grundsätzlich völlig unterschiedliche Entitäten gesehen wurden. Der Begriff „Dutch Hypothesis“ wurde auf dem Symposium „Bronchitis III“ von Fletcher geprägt - im Unterschied zur „British Hypothesis“ [2]. Fletcher selbst war damals der Meinung, die Dutch Hypothesis sei näher an der Realität als die British Hypothesis: “We conclude that the 'Dutch' hypothesis of the development of airways obstruction is closer to the truth than the 'British' hypothesis but we have found no evidence of hyperreactivity of the asthmatic type in those subjects who are developing the condition [ COPD]. We do not know why they are more susceptible than others to the effect of smoking."

Die Forschung war in den nachfolgenden Jahrzehnten geprägt von einer Präzisierung der Unterschiede zwischen Asthma und COPD. Die Unterschiede wurden zeitweilig sehr pointiert, mit Auswirkungen insbesondere auf die Therapie, die sich zuvor bei Asthma und COPD weitgehend glich. Zeitweilig wurde in den Leitlinien z.B. der Einsatz von Glukokortikosteroiden in der Dauer- 


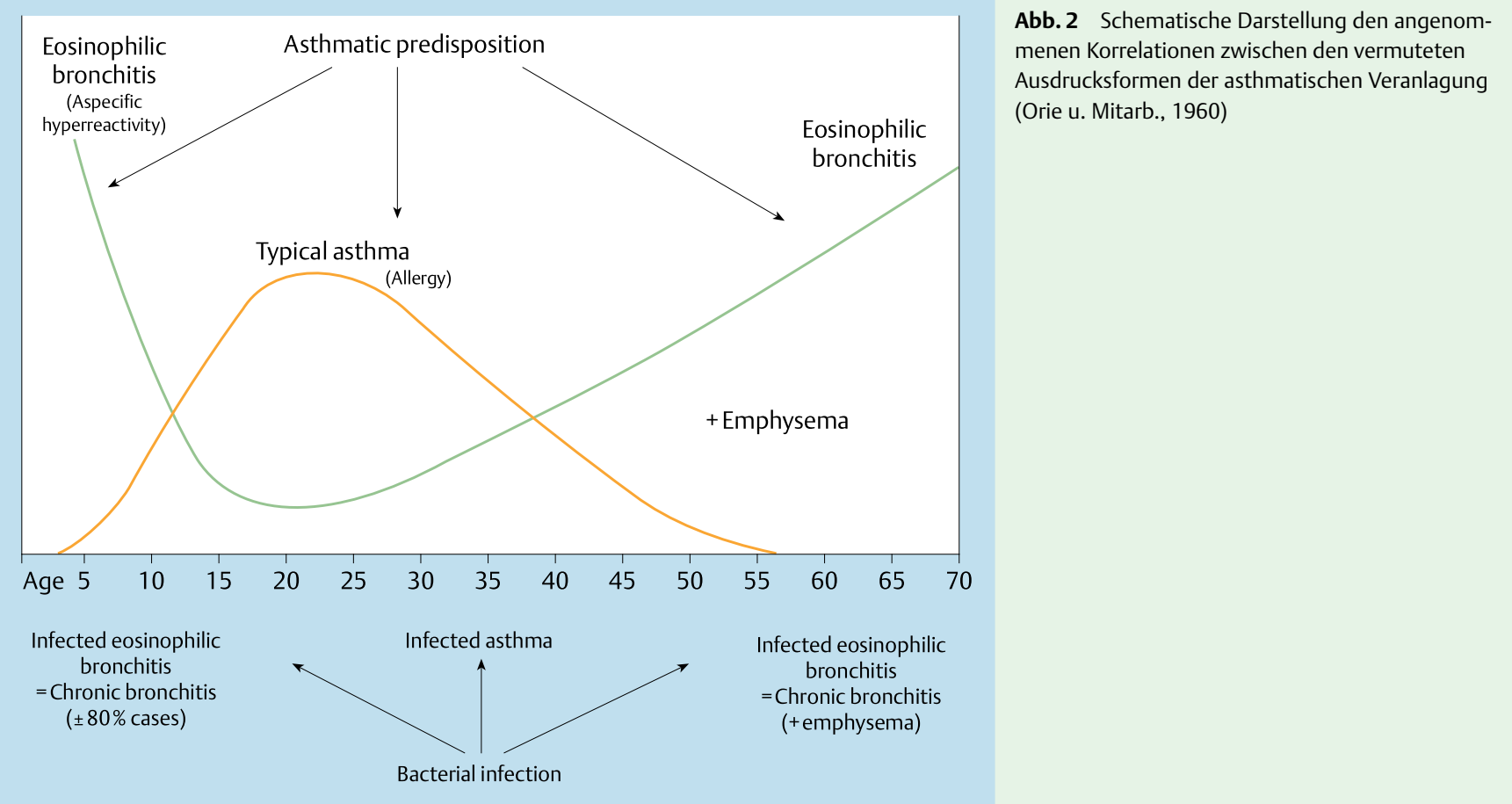

therapie der COPD weitgehend abgelehnt. Diese Auffassungen beruhten auf Studien bei älteren Erwachsenen, ohne Reversibilität der Atemwegsobstruktion unter Beta-Agonisten. Umgekehrt werden Therapiestudien bei Asthma im Erwachsenenalter bei jüngeren Nichtrauchern durchgeführt, die eine deutliche Reversibilität ihrer Obstruktion aufweisen. Diese Unterschiede der Einschlusskriterien mussten die Unterschiede von Asthma und COPD im Erwachsenenalter betonen - die häufigen Mischbilder wurden ausgeklammert. Erst in den letzten Jahren, nachdem Exazerbationen und damit wichtige Parameter des klinischen Alltags der obstruktiven Atemwegserkrankungen als Kriterium genutzt werden, sind Glukokortikosteroide auch bei COPD wieder auf dem Vormarsch, somit zumindest partiell in Übereinstimmung mit Ories Hypothese.

Nach heutigen Vorstellungen sind definitive Belege für die „Dutch Hypothesis“ nicht erbracht worden [7], und beide Entitäten weisen so erhebliche Unterschiede bezüglich Krankheitsverlauf, Symptomatik, Prognose und Therapierbarkeit auf, dass die Orie'schen Vorstellungen wenig Chancen haben, verifiziert zu werden. Orie selbst hat 2000 nochmals Stellung genommen [4]. Er betonte besonders, dass frühkindliche Infektionen die Basis für das spätere Auftreten einer COPD darstellen würden. Seine Nachfolger sehen in genetischen Studien die beste Möglichkeit, Gemeinsamkeiten und Unterschiede zu identifizieren [6].

Die „Dutch Hypothesis“ hat die wissenschaftliche Debatte über Asthma und COPD bis heute auf jeden Fall so außerordentlich belebt und die wissenschaftliche Entwicklung unseres Faches so nachhaltig beeinflusst, dass N.G.M. Orie ohne jede Einschränkung zu den herausragenden Vertretern unseres Faches gehört. Daher soll sein Lebenslauf und seine wissenschaftliche Bedeutung kurz dargestellt werden.

Orie wurde am 19.11.1914 in Ginneken en Bavel, heute Breda/ Niederlande geboren. Das Studium der Medizin absolvierte er bis 1939 in Utrecht [1]. Seine ärztliche Tätigkeit begann er im St. Joseph-Krankenhaus in Eindhoven, wo sein Interesse an Lungenkrankheiten geweckt wurde. Den größten Teil seines Lebens hat er in Westfriesland verbracht. 1945 erhielt er eine Internisti- sche Assistenzarztstelle in Groningen, wo er seither tätig war. 1946 wurde er an der Reichsuniversität Utrecht promoviert mit einer pneumologischen Arbeit („Over het voorkomen en de beteekenis van gisten in den Luchtwegen“). 1949 wurde auf seine Initiative in Groningen eine eigenständige Poliklinik für Lungenkrankheiten eingerichtet. 1955 wurde er zum Ordinarius für Pneumologie berufen. Dieses Amt übte er bis 1979 an der Reichsuniversität Groningen aus.- Er war Ehrendoktor der Reichsuniversität Leiden und Ritter des 1815 gegründeten Ordens des Niederländischen Löwens.

Prof. Orie wurde vor allem durch die „Bronchitis-Symposien“ in Groningen bekannt, die er 1960 begründete und die inzwischen von seinen Nachfolgern bis zum Bronchitis VII-Symposium 2003 weitergeführt wurden.

Der Verstorbene hat seit Jahrzehnten in Winsum, nahe Groningen, in einem ehemaligen Pastorat gelebt. Orie war nach dem Tod seiner ersten Frau 1984 ein zweites Mal verheiratet. Er hinlässt Kinder und Kindeskinder. - Orie hat auch nach seiner Emeritierung die wissenschaftliche Entwicklung verfolgt. Seine letzten wissenschaftlichen Publikationen stammen aus den Jahren 2000 und 2002, wiederum zur „Dutch Hypothesis“ [4,5].

Infolge seiner bedeutenden Beiträge zur COPD-Forschung erhielt Orie zahlreiche Ehrungen, unter anderem auch die Ehrenmitgliedschaft der Gesellschaft für Lungen- und Atmungsforschung in Bochum. Durch das jahrzehntelange gemeinsame Interesse an den vagalen Mechanismen der Atemwege und der Lungen bestanden persönliche Bindungen mit Prof. Ulmer und mit dem Unterzeichner - häufig hat Orie auch in den letzten Jahren mit einem brieflichen Gruß die Bochumer „Dezembertagung“ begleitet.

Prof. Orie war durch seine frühzeitig von der traditionellen - von der Tuberkulose geprägten - Pneumologie abweichenden Konzepte und ebenso auch durch seine herausragende Persönlichkeit einer der wichtigsten klinischen Pneumologen der zweiten Hälfte des vergangenen Jahrhunderts. Er verdient das bleibende Angedenken der Pneumologen weltweit. 


\section{Literatur}

1 Astma Fonds 2006.. Prof. Orie overleden www.astmafonds.nl

2 Fletcher CM, Peto R, Speizer FS et al. A follow-up study of the natural history of obstructive bronchitis. In: Orie NGM, van der Lende R (Hrsg): Bronchitis III. Assen/Niederlande: Royal Vangorcum, 1970: $103-116$

3 Orie NGM, Sluiter HJ, de Vries K et al. The host factor in bronchitis. In Orie NGM, Sluiter HJ (Hrsg). Bronchitis. An International Symposium 27-29 April 1960. Assen/Netherlands: Royal Vangorcum, 1961: 4359
4 Orie NGM. The Dutch Hypothesis. Chest 2000; 117: 299S

5 Orie NG. [The relationship between asthma and chronic obstructive and restrictive pulmonary diseases; lessons from the past century]. Ned Tijdschr Geneeskd 2002; 146: 1504-1508

6 Postma DS, Boezen HM. Rationale for the Dutch Hypothesis. Allergy and Airway Hyperresponsiveness as Genetic Factors and Their Interaction With Environment in the Development of Asthma and COPD. Chest 2004; 126: 96S-104S

7 Sluiter HJ, Koeter GH, de Monchy JG et al. The Dutch hypothesis (chronic non-specific lung disease) revisited. Eur Respir J 1991; 4: 479-489 